\title{
Improved Pulmonary Function in Chronic Quadriplegics after Pulmonary Therapy and Arm Ergometry
}

\author{
J. Walker, MD, PhD, M. Cooney, PhD, S. Norton, MA
}

The Walker Institute, 881 Alma Real Drive, Suite 110, Pacific Palisades, CA 90272, USA.

\begin{abstract}
Summary
Quadriplegics suffer from restrictive lung disease due to neural damage, spasticity, and prolonged immobilisation. These studies were undertaken to see whether respiratory therapy can improve expiratory capacity in quadriplegics. Fifteen patients with chronic quadriplegia participated in a programme of pulmonary therapy and resistance exercise for 7 to 12 weeks. Pulmonary therapy consisted of incentive spirometry for 15 minutes a day 3 to 5 days per week. Resistance exercise consisted of pedalling an arm ergometer up to 30 minutes three times a week.

Forced vital capacity was measured with the subject in a wheelchair wearing a nose clip. The volume of expired air (max $\dot{V} e)$ was as determined with a paramagnetic analyser during arm ergometry. The volume of expired air was divided by the number of breaths, giving the volume of expired air per breath $(\dot{V} e)$. The results indicated that a combination of incentive spirometry and arm ergometry improves vital capacity and increased the maximum volume of expired air during exercise. These methods are non-invasive, and such modalities should constitute part of the rehabilitation of patients with neuromuscular disease.
\end{abstract}

Key words: Spinal cord injury; Quadriplegia; Pulmonary function.

Respiratory complications are the primary cause of death in patients with chronic traumatic quadaperesis (Silver and Gibbon, 1968) and account for $40 \%$ of complications in all spinal cord injured (Cowell et al., 1986). In normal subjects, breathing occurs primarily through the use of the intercostal muscles and the diaphragm (Bergofsky, 1964). In quadriplegics, however, the intercostal muscles are paralysed, and ventilation occurs primarily through the use of the diaphragm (Bergofsky, 1964) and accessory muscles of the neck and shoulders (Guttmann, 1976; De Troyer et al., 1986). In general, the higher the level of the lesion, the more compromised the pulmonary function. Pulmonary capacity in quadriplegics may also be restricted because of spasticity, chronic immobility and diaphragmatic fatigue (Fugl-Meyer, 1971; Roussos and Macklem, 1977). Respiratory infections and atelectasis also increase the work of breathing (Bergofsky, 1964). 
Table Profile of subjects participating in the experiment according to age, sex, and level of injury

\begin{tabular}{ccccc}
\hline Level & Sex & Age & VC 1 ml $(\%)$ & VC 2 ml (\%) \\
\hline C5-6 & F & 26 & $2500(70)$ & $3400(96)$ \\
C5-6 & M & 18 & $4000(84)$ & $4800(101)$ \\
C4-5 & M & 17 & $1400(42)$ & $1600(49)$ \\
C6-7 & M & 32 & $2700(59)$ & $3000(66)$ \\
C5-6 & F & 24 & $2550(59)$ & $2700(63)$ \\
C5-6 & M & 29 & $2100(62)$ & $2900(85)$ \\
C5-6 & M & 19 & $2700(59)$ & $3500(77)$ \\
C6-7 & M & 25 & $3750(78)$ & $3900(82)$ \\
C4-5 & M & 17 & $1400(31)$ & $2000(44)$ \\
C6-7 & M & 27 & $2100(51)$ & $3000(71)$ \\
C5-6 & M 8 & 20 & $3400(71)$ & $3900(82)$ \\
C4-5 & $M$ & 32 & $2900(63)$ & $4200(92)$ \\
C5-6 & M & 26 & $2350(53)$ & $3000(70)$ \\
C6-7 & M & 33 & $2000(59)$ & $2800(82)$ \\
\hline
\end{tabular}

Forced vital capacity before participation in the experiment (VC 1) and after participation (VC 2). All subjects had sustained spinal trauma at least 2 years previously. Numbers in parenthesis indicate per cent of predicted VC while sitting.

There are no guidelines for providing respiratory care for the spinal cord injured in the United States (Clough, 1986) and respiratory care is not commonly provided for chronic spinal cord injured patients. Traditional therapeutic modalities include intermittent positive pressure breathing (IPPB), chest stretching, and assisted manual coughing. IPPB does not decrease the work of breathing patients with respiratory muscle weakness (De Troyer et al., 1981). Chest stretching and manually assisted coughing eliminate secretions but do not affect ventilatory muscle function per se. To our knowledge, there are no studies documenting the efficacy of incentive spirometry in improving ventilation in quadriplegics.

These results may provide the rational basis for the establishment of a protocol for improving the ventilation of subjects with chronic spinal cord injury. The advantages of the procedure are that it produces rapid improvement, it is non-invasive, inexpensive, and can be administered in any setting, even at home. We believe that all rehabilitation settings should use this type of pulmonary therapy in patients with neuromuscular disease.

\section{Methods}

After a medical screening which consisted of complete physical examination, routine laboratory tests including urinalysis, 12 lead electrocardiogram, and cardiovascular stress test, 15 non-smoking subjects with chronic quadriplegia participated in a programme of pulmonary therapy and resistance exercise for 7 to 12 weeks. Subjects were 15 incomplete quadriplegics who participated in the experiment. Except for 2 subjects who had received incentive spirometry for 2 weeks during the acute phase of rehabilitation, none of the subjects had received prior pulmonary therapy. Subject characteristics are presented in the Table. Pulmonary therapy consisted of incentive spirometry for 15 minutes a day, 3 to 5 days per week (Pollock et al., 1974. Incentive spirometry was performed by 
exhaling into a spirometer, which consisted of two adjacent cylinders containing water. Through expiration, water was transferred from one cylinder to another cylinder (Spiroflow Blow Bottle, Cheseborough Ponds Inc., Greenwich, Conn) and subjects were monitored by a physical therapist for fatigue or dizziness.

Resistance exercise consisted of arm ergometry (Monark, Redmond, Washington) for up to 30 minutes three times a week with constant monitoring vital signs, and electrocardiocardiograms. Exercise was discontinued if the subject felt dizzy, nauseated or exhausted, thus this was a symptom-limited exercise test. With increasing tolerance, the resistance on the arm ergometer was increased. Work was calculated as resistance of the wheel (in kilograms) times revolutions per minute times distance (metres/revolution) and expressed as watts.

Forced vital capacity was measured after maximal inspiration three times while the subject sat in a wheelchair wearing a noseclip. The vital capacity was taken as the best score although the within subject variability was only $100 \mathrm{cc}$. Forced vital capacity was measured before participation in the experiment and at the end. The volume of expired ( $\dot{\mathrm{Ve}}$ ) air was determined with the ergooxyscreen paramagnetic analyser (Erich Yeager, Inc. Rockford, Illinois) on a minute by minute basis as was the number of breaths per minute ( $\max \mathrm{Ve}$ ). As the name implies, max $\dot{V}$ e was obtained as the maximum ventilatory effort during a trial, regardless of which minute the effort occurred during arm ergometry. The maximum volume of expired air was divided by the numbers of breaths (also recorded on the analyser), giving the volume of expired air per breath per minute $(\dot{V} t)$. For all calculations, max $\dot{V}$ e and max $\dot{V} t$ were taken.

\section{Results}

Paired comparison t-tests using the Bonferroni correction for multiple comparisons (Glantz, 1981) were used to assess the possible effects of treatment on vital capacity, $\max \dot{\mathrm{V} e}$, work output, and volume of expired air (expressed as $\mathrm{cc} / \mathrm{min}$ ). The Bonferroni t-test represents the most conservative parametric test for performing multiple comparisons. If one analysed a set of data with four t-tests using the $5 \%$ critical value for concluding there is a difference, there is a $4(5)=20 \%$ chance of finding a difference when it does not really exist. The Bonferroni inequality corrects for performing multiple comparisons by using a higher cut-off for alpha. The greater the number of comparisons, the more stringent the cut-off point for alpha, so that the error rate is controlled (Glantz, 1981).

The results of the forced vital capacity are presented in the Table. All 15 of the 15 subjects demonstrated an increase in vital capacity. The mean vital capacity at the end of the experiment was $2500 \mathrm{ml}( \pm 765)$. The mean vital capacity at the end of the experiment was $3260 \mathrm{ml}( \pm 855)$, showing an average increase of $23.6 \%$. These results were significant $(\mathrm{t}=3.74, \mathrm{df}=14, \mathrm{p}<\cdot 02)$ using the correction for multiple comparisons.

The maximum volume of exhalation $(\max \dot{V} \mathrm{e})$ was $20.6 \mathrm{l} / \mathrm{min}(\mathrm{t} \lambda=7.7 \mathrm{~S} . \mathrm{D})$ before the experiment. After the experiment, $\max$ Ve rose to 34.9 ( \pm 13.3 S.D). The average increase in $\dot{\mathrm{Ve}}$ is $69.6 \%$ (Fig. 1), and all 15 of the 15 subjects demonstrated the increase in maximum volume of exhalation. The results were 


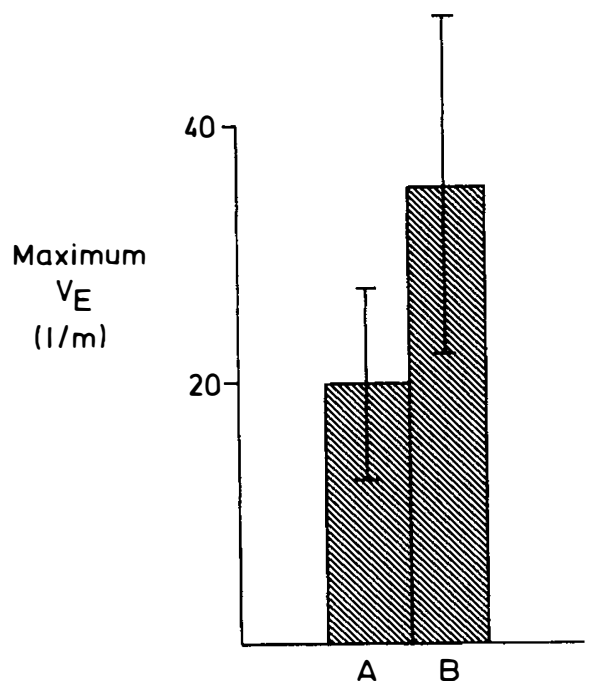

Figure 1 Maximum minute ventilation of quadriplegics during arm ergometry ( $1 /$ minute) before participation in pulmonary therapy (A) and after participation (B). All of the subjects demonstrated an increase. Bars indicate standard deviation.

also significant $(\mathrm{t}=3.793, \mathrm{df}=14, \mathrm{p}<\cdot 01)$. Maximum exercise power rose from 17.4 watts $( \pm 2.4$ S.D) to 28.6 watts $( \pm 4.9$ S.D) (Fig. 2). All 15 subjects demonstrated the increase $(\mathrm{t}=4 \cdot 22, \mathrm{df}=14, \mathrm{p}<\cdot 02)$.

The volume exhaled was calculated by dividing max Ve by breathing frequency during that 1 minute period. The results showed an increase in the volume expired expressed as cc/breath. The volume of this was $590 \cdot 3 \pm 180 \mathrm{cc}$ at the beginning of the experiment and rose to $850 \pm 280 \mathrm{cc}$ at the end of the experiment (Fig. 3). This represents a $43 \%$ improvement, and all of the subjects demonstrated this improvement $(\mathrm{t}=3 \cdot 74, \mathrm{df}=14, \mathrm{p}<\cdot 02)$.

\section{Discussion}

The present results indicate that a combination of arm ergometry and incentive spirometry increase ventilatory function in quadriplegics. Although expiratory muscle strength was not measured directly, the large increase in volume of expired air suggests but does not prove there was an increase in expiratory muscle strength. Inspiratory training also improves respiratory function in quadriplegia (Gross et al., 1980). The present method (arm ergometry) has the advantage of simultaneously providing patients with cardiovascular fitness training (Pollock et al., 1974). Increased endurance in our subjects is suggested by the observation that all demonstrated a large increase in work. We are presently studying the effect of arm ergometry on cardiovascular fitness in quadriplegics. Further work is being performed to determine whether incentive spirometry, arm ergometry, or the combination are responsible for the improvement.

The results, unfortunately, do not indicate which muscle groups are involved in maximum ventilatory expiration. Average expiration values for normals are in the range of $150 \mathrm{l} /$ minute. Expiration in quadriplegics is accomplished by 


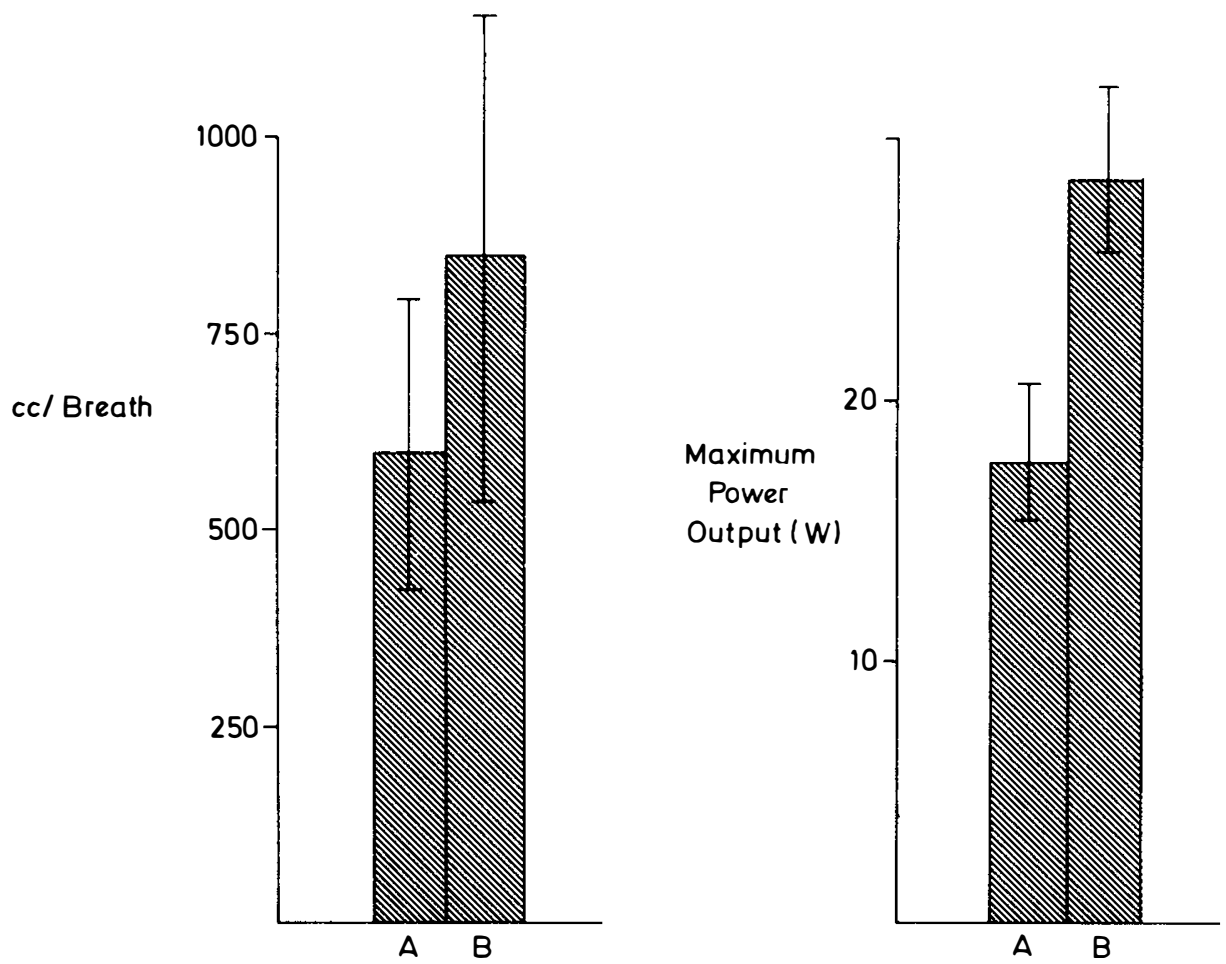

Figure 2 Maximum volume of expired air per breath of quadriplegics during arm ergometry before participation in pulmonary therapy (A) and after participation (B). All of the subjects demonstrated an increase. Bars indicate standard deviation.

Figure 3 Maximum power output (watts) of quadriplegics during arm ergometry before pulmonary therapy (A) and after participation in the experiment (B). All of the subjects demonstrated increase in exercise capacity. Bars indicate standard deviation.

the contraction of accessory muscles of the neck and shoulders as well as the diaphragm (Guttmann, 1975; De Troyer et al., 1986). It is unlikely that these muscles can account for the vigorous nature of expiration in these patients. Six of the 15 quadriplegics achieved a maximum $\dot{V} e$ of $40 \mathrm{l} /$ minute or greater (Walker and Cooney, 1987). Comroe (1974) states that at above $40 \mathrm{l} /$ minute, the abdominal muscles begin to contract. Since Comroe was addressing himself to respiration in able-bodied subjects, his comments do not necessarily apply to chronic quadriplegics. It would be interesting to discover which muscles are recruited in such high volume breathers.

\section{References}

BERGOFSKY EH 1964 Mechanism for respiratory insufficiency after cervical cord injury: A source of alveolar hypoventilation. Annals of Internal Medicine 61:435-447.

Clough P, Lindenauer D, Hayes M, et al. 1986 Guidelines for routine respiratory care of patients with spinal cord injury. Physical Therapy 66:1395-1402.

Comroe JH 1974 Respiratory Physiology. Year Book Medical Publishers, Chicago, pp 94-141.

COWELL LL, SQUIRES WG, RAVEN RB 1986 Benefits of aerobic exercise for the paraplegic: a brief review. Medicine and Science in Sports and Exercise 18:501-508.

De Troyer A, DeIsSER P 1981 The effects of intermittent positive pressure breathing on 
patients with respiratory muscle weakness. American Review of Respiratory Disease 124:132137.

FUGL-MEYER AR 1971 Effects of respirtory muscle paralysis in tetraplegic and paraplegic patients. Scandinavian fournal of Rehabilitation Medicine 3:141-150.

Giantz SA 1981 Primer of Biostatistics. McGraw-Hill, New York, p 87.

GROSS D, LADD HW, RILEY EJ, et al. 1980 The effect of training on strength and endurance of the diaphragm in quadriplegia. American fournal of Medicine 60:27-68.

GuTtMANN LG 1976 Spinal cord injuries: comprehensive management and research. Blackwell Scientific Publications.

LEITH DE, BRADLEY M 1976 Ventilatory muscle strength and endurance training. Fournal of Applied Physiology 41:508-516.

Pollock ML, Miller HS, Linnerud AC, et al. 1974 Arm pedaling as an endurance training regimen for the disabled. Archives of Physical Medicine and Rehabilitation 55:418-424.

Roussos CS, MACKLEM PT 1977 Diaphragmatic fatigue in man. Fournal of Applied Physiology: Respiratory, Environmental and Exercise Physiology 43:189-197.

SILVER JR, GibBon NO 1968 Prognosis in tetraplegia. British Medical fournal 4:79.

WALKER J, COONEY MM 1987. Improved respiratory function in quadriplegics after pulmonary therapy and arm ergometry. New England fournal of Medicine 316:486-487. 